Chapter 10

\title{
Fiber-Based High-Power Supercontinuum and Frequency Comb Generation
}

\author{
Qiang Hao, Tingting Liu and Heping Zeng \\ Additional information is available at the end of the chapter \\ http://dx.doi.org/10.5772/64209
}

\begin{abstract}
Ultrafast optics has been a rich research field, and picosecond/femtosecond pulsed laser sources seek many applications in both the areas of fundamental research and industrial life. Much attention has been attached to fiber lasers in recent decades as they offering various superiorities over their solid-state counterparts with compact size, low cost, and great stability due to the inherent stability and safety of the waveguide structures as well as high photoelectric conversion efficiency. Fiber-based sources of ultrashort and high-peak/high-average optical pulses have become extremely important for high-precision laser processing while sources whose carrier-envelop offset and repetition rate are stabilized can serve as laser combs with applications covering many research areas, such as precision spectroscopy, optical clock, and optical frequency metrology. For the application as laser combs, four parts as fiber laser, broadband supercontinuum, nonlinear power amplification, and repetition rate stabilization must be concerned. This chapter is intended to give a brief introduction about the achievement of the four technologies mentioned above with different experimental setups, recently developed such as divided-pulse amplification (DPA) in emphasize. Moreover, detailed descriptions of the experimental constructions as well as theoretical analyses about the phenomena they produced are also involved.
\end{abstract}

Keywords: fiber lasers, divided-pulse nonlinear amplification, four-wave mixing, frequency stabilized

\section{Introduction}

Ultrafast laser sources and their applications such as high-power supercontinuum and frequency comb have gained much attention in recent decades [1-7]. High-power fiber lasers 
spur a rapid growth of industrial applications including laser cutting, laser marking, and so on [8]. Moreover, supercontinuum and frequency comb are considered as the breakthrough of laser field for their applications covering precision spectroscopy, astronomical observations, and optical frequency metrology $[9,10]$. This chapter is intended to describe, from experimental point of view, the ultrashort pulse laser oscillators, high-power nonlinear fiber amplifiers, supercontinuum, and frequency combs. Section 2 shows the performance of two types of mode-locked lasers. The first one consisting of bulk and fiber optical components is mode-locked via nonlinear polarization rotation (NPR) mechanism at $1.03 \mu \mathrm{m}$. The other one, operating at $1.55 \mu \mathrm{m}$, is mode-locked by nonlinear amplified loop mirror (NALM) with polarization-maintaining (PM) fiber components in order to overcome environmental perturbation and thus maintain long-term operation. Section 3 introduces a practical method (spectral tailoring), which facilitates supercontinuum generation in single-mode fiber amplifier at $1.03 \mu \mathrm{m}$ with a few picosecond laser pulses. The second part in this section introduced broadband supercontinuum generation (from 950 to $2200 \mathrm{~nm}$ ) by injecting pulses with 72-fs temporal duration, $150-\mathrm{mW}$ average power, and 60-MHz repetition rate at $1560 \mathrm{~nm}$ into 20-cm-long PM-HNLF. Section 4 gives a brief introduction of divided-pulse amplification (DPA). To generate transform-limited pulse at $1.55 \mu \mathrm{m}$, DPA with polarized pulse duplicating was employed to overcome the gain narrowing effect and control the nonlinear spectral broadening in anomalous dispersion Er-fiber amplifier. As high as $500-\mathrm{mW}$ average power at $1560 \mathrm{~nm}$ is achieved by $\times 8$ replicas. Moreover, the highest frequency-doubling conversion efficiency reached $56.3 \%$ by using a periodically poled lithium niobate (PPLN) crystal at room temperature. Section 5 discusses an all-optical control method via resonantly enhanced optical nonlinearity (or pump-induced refractive index change, RIC) for highprecision repetition rate stabilization. The standard deviation (SD) of repetition rate can be reduced to a record level of $<100 \mu \mathrm{Hz}$ by using the RIC method in a PM figure-eight laser cavity.

\section{Fiber laser}

Fiber lasers offer several practical advantages, such as excellent spatial-mode quality, effective heat dissipation, and flexible optical path and, recently, are becoming attractive laser sources in both scientific researches and industry applications. Especially, mode-locked fiber lasers with ultrashort pulse duration and high-repetition rate have attracted a lot of attention for their applications in optical sensing, optical communication, optical metrology, and biomedical imaging and processing $[11,12]$. Therefore, various femtosecond/picosecond mode-locked lasers have been constructed and developed. As mode-locked lasers are often affected by environmental perturbations (mechanical vibration and temperature fluctuation), robust and stable oscillator with compact design are urgently needed. In this section, we present a compact femtosecond fiber laser at $1.03 \mu \mathrm{m}$ by using integrated fiber optical components. The shortest dechirped pulse duration reaches 81 fs for a net cavity dispersion value close to $-0.001 \mathrm{ps}^{2}$. Another part of this section described a self-started Er-doped laser oscillator, which is mode- 
locked by NALM with PM-fiber configuration. By optimizing the net dispersion, the buildup time can be reduced from $8 \mathrm{~min}$ to $\sim \mathrm{ms}$ order of magnitude.

\subsection{Operation regime of mode-locked lasers}

As well known, the main features of mode-locked fiber laser depend on the pulse evolution process, which is relevant to the group-velocity dispersion (GVD) and the nonlinearity in optical fibers. According to the net intra-cavity dispersion, the pulse-shaping process can be roughly distinguished into the four different regimes, such as soliton regime, stretch-pulse regime, parabolic-pulse regime, and giant-chirp pulse regime, corresponding to all-anomalous dispersion, normal-anomalous dispersion, all-normal dispersion, and large-normal dispersion, respectively. Due to the equilibrium between Kerr nonlinearity and GVD, pulses that propagate in all-anomalous dispersion laser cavity keep unchanged in the form of fundamental soliton [13, 14]. While in dispersion-managed laser cavities, the negative dispersion is compensated by positive dispersion and thus stretch pulse forms. When the net cavity dispersion is optimized to zero, significant variations on pulse duration could be observed $[15,16]$. As pulse operates in the all-normal dispersion regime, where laser gain, selfphase modulation, and dispersion co-effect, spectral/temporal filtering effects force linear chirping in the pulse, so that similariton forms [17-19]. While in ultra-long laser cavity, giantchirped oscillating can be realized with ultralow repetition rate but at high-pulse energy [2023].

\subsection{NPR mode locking at $1.03 \mu \mathrm{m}$}

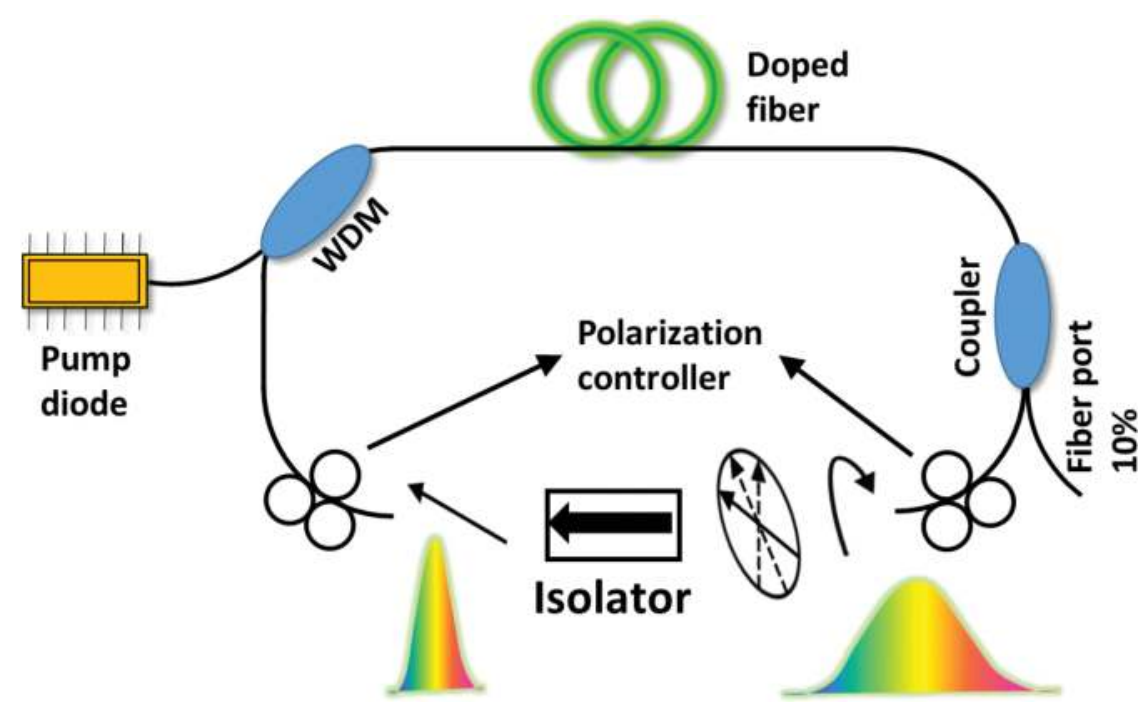

Figure 1. Schematic diagram of a passively mode-locked fiber laser via nonlinear polarization rotation mechanism. 
In this section, we designed a compact ultra-fast $\mathrm{Yb}$-doped fiber laser with integrated optical components. By integrating wavelength division multiplexer and optical isolator with collimators, the fiber loop was simplified. Self-started mode-locking could be realized by setting appropriate polarization angle of four intra-cavity wave plates. Due to the normal dispersion of fiber at $1.0 \mu \mathrm{m}$, transmission grating pair with $1250 \mathrm{l} / \mathrm{mm}$ was used to provide adjustable anomalous dispersion. As a result, $81-\mathrm{fs}$ temporal duration with $65-\mathrm{MHz}$ repetition rate and 0.5-nJ pulse energy was produced.

The mode-locking procedure can be explained in Figure 1. Two polarization controllers and a polarization-sensitive isolator (PSI) are used as the key elements for mode-locking. This combination acts as a virtual saturable absorber, which can absorb the low-intensity tail of pulse and transmit high-intensity part such that the pulse could be shortened. The pulse with linear polarization changes to elliptical polarization by twisting the polarization controller. As mentioned, self-phase modulation (SPM) or cross-phase modulation (XPM) can arouse energy coupling between two orthogonal polarizations. Moreover, serious nonlinear polarization rotation is produced by the high gain in the active fiber. Finally, another polarization controller is used to modify the polarization state to facilitate the central part of the pulse getting through the PSI [24].

In our experiment, a Yb-doped fiber laser shown in Figure 2(a) was firstly constructed without dispersion compensation elements. Three intra-cavity wave plates including two quarterwave plates, QWP1 and QWP2, and one half-wave plate, HWP, were set with appropriate polarization angles to realize self-started mode-locking. The pigtails of fiber components are Hi1060 fiber with GVD of $\sim 26 \mathrm{fs}^{2} / \mathrm{mm}$ and TOD of $\sim 41 \mathrm{fs}^{3} / \mathrm{mm}$, while the GVD for the active fiber is $39 \mathrm{fs}^{2} / \mathrm{mm}$. The repetition rate and pulse duration were measured to be $70 \mathrm{MHz}$ and 13 ps, respectively. By external-cavity dechirping, the pulse can be compressed to $170-\mathrm{fs}$ duration, but with obvious pedestal. The autocorrelation of pulse before and after dechirping is shown in Figure 3(a) and (b), respectively.

(a)

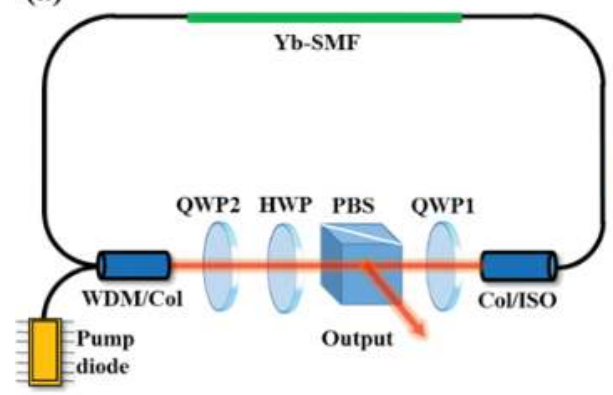

(b)

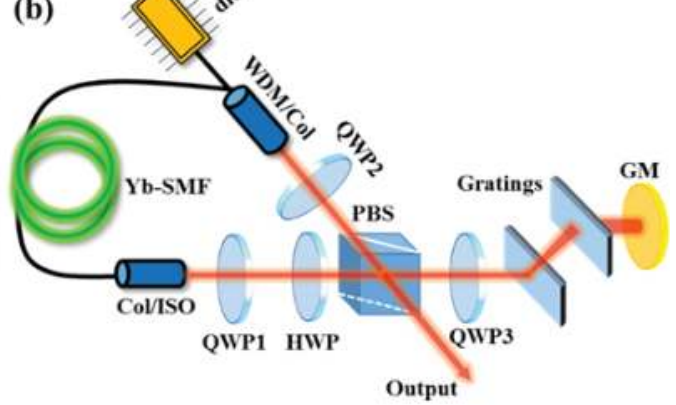

Figure 2. Structure of ultrafast $\mathrm{Yb}$-doped fiber lasers without (a) and with (b) dispersion compensation. Pump diode: 400-mW laser diode at $976 \mathrm{~nm}$; Yb-SMF: Yb-doped single-mode fiber; WDM/Col: the device combines WDM and colli- 
mator; Col/ISO: the device combines collimator and isolator; QWP1, QWP2, and QWP3: quarter-wave plate; HWP: half-wave plate; PBS: polarization beam splitter; GM: a gold-coated mirror.
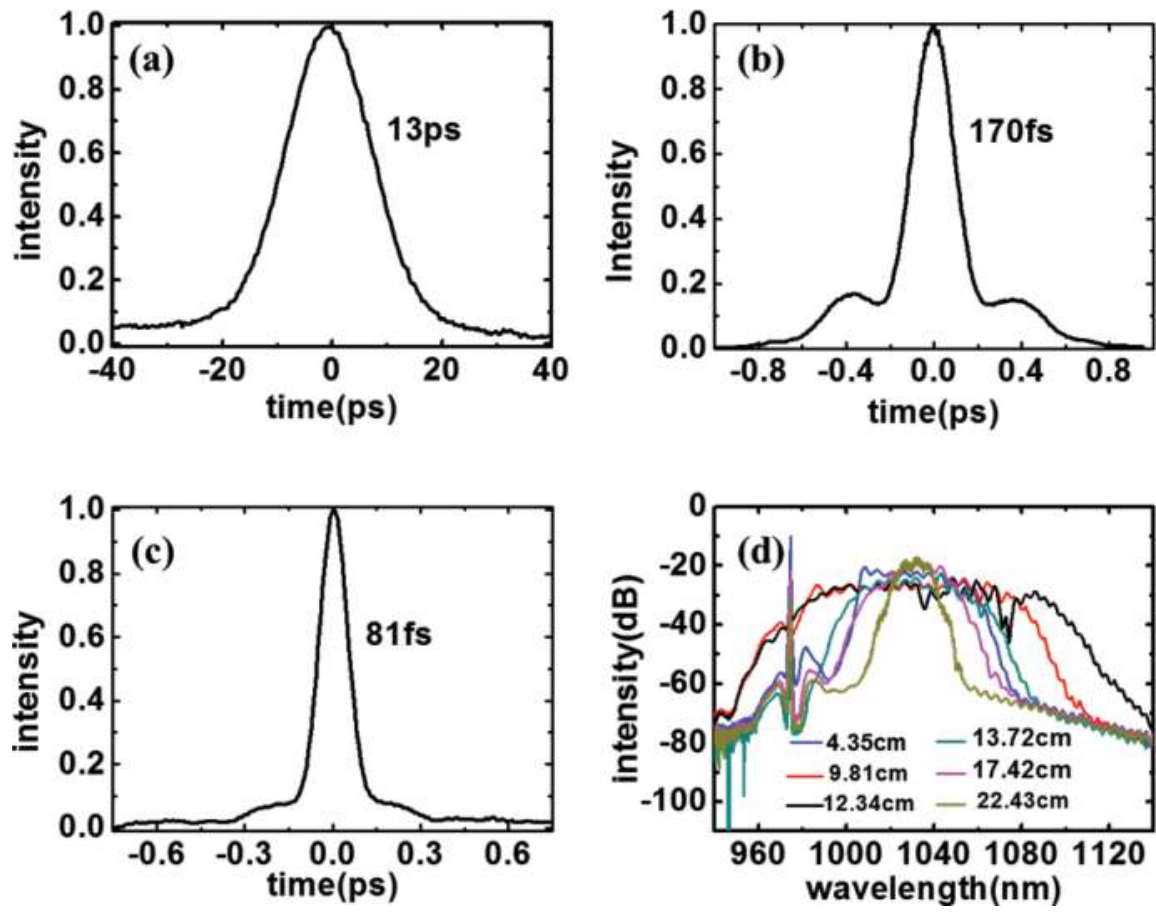

Figure 3. Autocorrelation trace of chirped pulses generated by picosecond fiber laser (a) and femtosecond fiber laser (c), (b) dechirped pulse of (a); (d) spectra generated by femtosecond fiber laser with different distances between gratings.

Secondly, a transmission grating pairs was used to manage the intra-cavity dispersion of the $\mathrm{Yb}$-fiber laser, as shown in Figure 2(b). The quarter-wave plate, QWP3, was used to impose $90^{\circ}$ polarization rotation on laser pulses by double-passing the grating pairs. Soliton, stretchpulse, and all-normal dispersion regime can be achieved by optimizing the distance between gratings. Figure 3(d) compares the various spectral shapes with different grating separations. As shown in Figure 3(c), the shortest pulse duration was measured to be $81 \mathrm{fs}$. The black curve in Figure 3(d) represents the broadest spectra with a 10-dB bandwidth of $100 \mathrm{~nm}$. The uncompensated phase was mainly caused by the accumulated high-order dispersion in fibers as well as intra- and extra-cavity grating pairs.

\subsection{Polarization-maintaining figure-eight fiber laser at $1.55 \mu \mathrm{m}$}

Compact size, low cost, and free maintenance fiber laser at $1.55 \mu \mathrm{m}$ are desirable in many applications, such as eye surgery, Terahertz generation, and precision spectroscopy [25-28]. The standard repetition rate of commercial available fiber laser is typically $80 \mathrm{MHz}$ with an 
optional design from 20 to $250 \mathrm{MHz}$. In order to combine both high pulse energy and high average power, a 10- $\mathrm{MHz}$ repetition rate is the best choice for applications. When the repetition rate is lower than $10 \mathrm{MHz}$, a pulse picker has to be used between the laser oscillator and the succeeding amplifier.

In this section, we introduce a PM figure-eight laser cavity which is the best option for oscillator operated at $10-\mathrm{MHz}$ repetition rate. Figure 4(a) shows the experimental setup of the figureeight laser cavity. The linear loop comprises of a 980/1550 nm wavelength division multiplexer, a segment of Er-doped fiber (PM-ESF-7/125, Nufern), an isolator, a 2-nm bandpass filter at $1550 \mathrm{~nm}$, and an output coupler CP2 with a splitting ratio of 20:80. The active gain applied in the linear loop is to compensate the cavity loses and facilitate self-started modelocking. The band-pass filter is used to block longer wavelength (Raman self-frequency shift) and reduce the temporal width of the pulse to be self-consistent. Pulses from the linear loop are coupled into NALM via CP1 with a splitting ratio of 45:55.

Over-pump with three LDs was applied to provide enough power for self-started modelocking. Interestingly, the buildup time of mode-locking was found to be closely related to the net cavity dispersion. When the net dispersion was set to $-0.115 \mathrm{ps}^{2}$, as long as 8-min buildup time was observed. After optimizing the net dispersion to about $-0.062 \mathrm{ps}^{2}$, the time dramatically decreased to $\sim$ ms of magnitude.
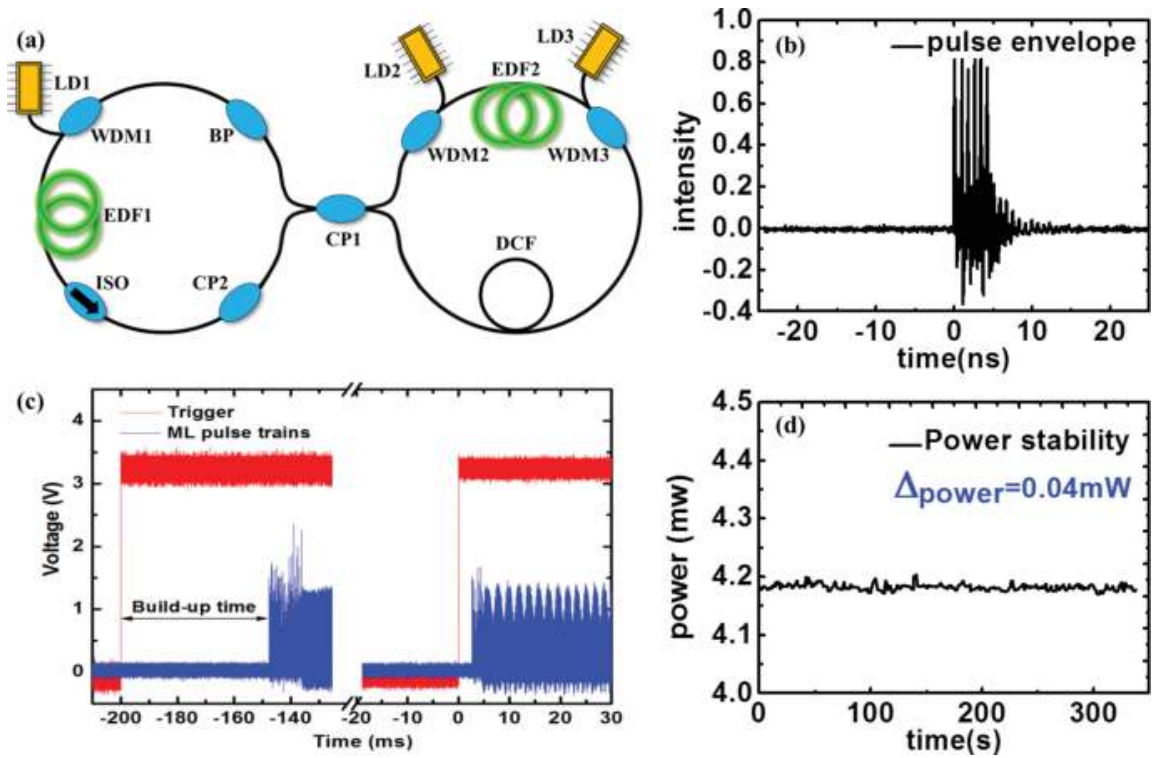

Figure 4. (a) Schematic of a polarization-maintaining figure-eight erbium-doped fiber laser. WDM1, WDM2, and WDM3: 980/1550 nm wavelength division multiplexers; ISO: isolator; EDF1 and EDF2: erbium-doped fiber; BP: 2-nm bandpass filter at $1550 \mathrm{~nm}$; DCF: dispersion compensation fiber; CP1 and CP2: $1550 \mathrm{~nm}$ coupler with splitting ratio of 45:55 and 20:80. (b) The initial pulse that polarization-maintaining figure-eight erbium-doped fiber laser generated. (c) The buildup time measured by an oscilloscope. (d) The measurement of power stabilization once mode-locked. 
Furthermore, we recorded the mode-locked pulse trains triggered by a square wave with 5$\mathrm{Hz}$ modulation frequency which is simultaneously used to drive LD3. Figure 4(c) shows two adjacent periods with 50-ms pump duration, and the corresponding buildup time was measured to be 53 and $6 \mathrm{~ms}$, respectively, exhibiting certain randomness. From experimental results point of view, the mode-locking buildup time is a random value in a certain range, which is related to the net cavity dispersion.

Interestingly, multiple-pulse operation was observed as the mode-locking is established, as shown in Figure 4(b). Peak power clamping effect originating from sagnac mechanism resulted in the formation of pulse bunching [29]. Stable single pulse could be obtained by decreasing the pump power. In single-pulse regime, the 5-min power stability was measured to be $0.26 \%$, as shown in the inset of Figure 4(d).

\section{Broadband supercontinuum}

Recent years, supercontinuum generation (SC) has attracted much attention for its applications in optical coherence tomography, stimulated emission depletion microscopy, dense wavelength-division-multiplexing (DWDM) optical networks, and frequency comb generation [30-33]. In this section, several nonlinear optical effects such as SPM, XPM, four-wave mixing (FWM), and stimulated Roman scattering (SRS) that facilitate SC generation would be firstly discussed. Secondly, spectral filtering method is demonstrated to be an effective way for broadband supercontinuum generation in picosecond region [34]. By spectral filtering, a linear-chirped picosecond pulse with a 1-nm bandwidth filter installed between two Yb-doped single-mode preamplifiers, pulse shortening, and high peak power is achieved, so that an octave-spanning SC with bandwidth of $650 \mathrm{~nm}$ from 750 to $1400 \mathrm{~nm}$ and 10-dB peak-to-peak flatness was obtained at an output average power of $190 \mathrm{~mW}$. Thirdly, SC covering from 950 to $2200 \mathrm{~nm}$ is generated in a 20-cm-long PM HNLF by injecting 72-fs pulse with $150-\mathrm{mW}$ average power and $60-\mathrm{MHz}$ repetition rate at $1.56 \mu \mathrm{m}$. Furthermore, an inline f-2f interferometer, including a PPLN for frequency doubling and a PM-fiber delay line, is used to generate carrier-envelop offset signal $\left(f_{\text {ceo }}\right)$.

\subsection{Nonlinear effects in optical fibers}

Most of nonlinear effects in optical fibers attribute to nonlinear refraction, which refer to the intensity dependence of the refractive index. Especially, the lowest order nonlinear effects in optical fibers originate from the third-order susceptibility $\chi^{(3)}$, which governs the four-wave mixing, Raman effect, third-harmonic generation, and polarization properties [24].

This section does not thoroughly focus on the discussion of theoretical issues. In simple, the refractive index of the optical fiber can be described by the following equation

$$
n=n_{0}+n_{1}|E(t)|^{2}
$$


where $n_{0}$ is the linear part and $n_{1}|E(t)|^{2}$ is the nonlinear part.

An interesting phenomenon of the intensity dependence of the refractive index change in optical fiber occurs through SPM. When the input pulse is of low intensity, the corresponding refractive index remains a constant $n_{0}$. As the input pulse increases, the corresponding refractive index becomes nonlinear change with power intensity $I$. Hence, an additional phase shift is produced:

$$
\delta \varphi(\mathrm{t}) \propto|E(t)|^{2}
$$

This can be understood as an instantaneous optical frequency change from its central frequency:

$$
\delta \omega(\mathrm{t})=-\frac{\partial}{\partial t} \delta \varphi(t)
$$

Therefore, new spectral components are generated and time dependent frequency chirping is produced.

Another most widely studied nonlinear effect is XPM, which leads to asymmetric spectral and temporal changes for two co-propagating optical fields with different wavelength or orthogonally polarization. The contribution of the nonlinear phase shift induced by XPM is twice that of SPM. Therefore, the nonlinear part $\Delta n_{\mathrm{j}}$ induced by the third-order nonlinear effects is given by $(j=1,2)$

$$
\Delta n_{j} \approx n_{2}\left(\left|E_{j}\right|^{2}+2\left|E_{3-j}\right|^{2}\right)
$$

Eq. (4) shows the refractive index of the optical media seen by an optical field inside a singlemode fiber depends not only on the intensity of that field but also on the intensity of the other co-propagating fields [35]. As the optical field propagates inside the fiber, an intensitydependent nonlinear phase shift shows up

$$
\phi_{j}^{N L}(z)=n_{2}\left(\omega_{j} / c\right)\left(\left|E_{j}\right|^{2}+2\left|E_{3-j}\right|^{2}\right) z
$$

The first term is related to SPM while the second term is related to XPM.

Stimulated Raman scattering (SRS) is an important nonlinear process that can produce redshifted spectral components. Once the spectrum of the input pulse is broad enough, the Raman gain can amplify the long-wavelength components of the pulse with the short-wavelength components acting as pumps, and the energy appears red-shifted. The longer the propagat- 
ing fiber, the more red-shifted spectral components can be generated. The red-shifted components are called Stokes wave. The initial growth of the Stokes wave can be described by

$$
\frac{d I_{s}}{d z}=g_{R} I_{p} I_{s}
$$

where $I_{\mathrm{s}}$ is the Stokes wave intensity, $I_{\mathrm{p}}$ is the pump-wave intensity, and $g_{\mathrm{R}}$ is the Raman-gain coefficient, which is related to the cross section of spontaneous Raman scattering.

The Raman-gain coefficient $g_{\mathrm{R}}(\Omega)$ is the most important factor to describe SRS. $\Omega$ represents the frequency difference between the pump wave $\omega_{\mathrm{p}}$ and Stokes wave $\omega_{\mathrm{s}}$. In the case of silica fibers, the Raman-gain spectrum is found to be very broad, extending up to approximately $40 \mathrm{THz}$. Assuming the pump wavelength is $1.5 \mu \mathrm{m}$ and, peak gain is $g_{\mathrm{R}}=6 \times 10^{-14} \mathrm{~m} / \mathrm{W}$, the frequency downshift can be calculated to be $13.2 \mathrm{THz}$.

When supercontinuum is generating in an optical fiber, the SPM, XPM, and SRS are always accompanied by FWM. In optical fibers, FWM transfers energy from pump wave $\left(\omega_{\mathrm{p}}\right)$ to two other waves in frequency domain, blue-shifted (anti-Stokes wave, $\omega_{\text {as }}$ ) and red-shifted (Stokes wave, $\left.\omega_{\mathrm{s}}\right)$. Once the phase-matching condition $\Delta k=2 k\left(\omega_{\mathrm{p}}\right)-k\left(\omega_{\mathrm{s}}\right)-k\left(\omega_{\mathrm{as}}\right)=0$ is satisfied, the Stokes and anti-Stokes waves can be amplified from noise or an incident signal at $\omega_{\mathrm{s}}$ or $\omega_{\mathrm{as}}$ respectively [36, 37]. Therefore, FWM process is used to produce spectral sidebands for supercontinuum generation.

\subsection{Supercontinuum generation}

$\mathrm{SC}$ is a powerful laser sources for many applications, such as nonlinear microscopy, optical coherence tomography, and frequency metrology [38-40]. Nowadays, more than one octave SC can be easily generated with a length of PCF, and the average power can reach tens of Watts [41, 42]. When ultrashort optical pulses propagate through a PCF fiber, the combination of SPM, XPM, SRS, and FWM is responsible for the spectral broadening. Generally speaking, the feature of SC depends on whether the incident laser wavelength $\lambda$ located is below, closed to, or above the Zero-dispersion wavelength $\lambda_{\mathrm{D}}$ of the PCF. In the anomalousdispersion regime $\left(\lambda>\lambda_{\mathrm{D}}\right)$ where $\beta_{2}<0$, soliton affects. If the $\lambda$ nearly coincides with $\lambda_{\mathrm{D}}, \beta_{2} \approx 0$, $\beta_{3}$ dominant and the phase-matching condition of FWM are approximately satisfied. While in the normal-dispersion regime, $\beta_{2}>0$, GVD and SPM effects dominant SC generation. From the time domain of view, SPM and soliton effects dominant SC generation for femtosecond (typically $<1$ ps) pump pulses, while FWM and SRS contribute to spectral broadening for tens of picoseconds pulses.

\subsubsection{Spectrally filtered seed for broadband supercontinuum generation in single-mode fiber amplifiers}

There are several methods to extend the SC spectrum. Considerable spectral broadening could be observed with high-power incident laser. High-average/high-peak powers facilitate $\mathrm{CW}$ and pulse SC generation [43-45]. Besides, the SC bandwidth could also be increased by tapering PCFs. A flat ( $3 \mathrm{~dB}$ ) spectrum from 395 to $850 \mathrm{~nm}$ was achieved in a tapered fiber with 
a continuously decreasing ZDW [46]. In this section, we demonstrate an effective method for broadband SC generation, which is valid in normal-dispersion fiber amplifiers. By spectral filtering of upchirped pulse at $1028 \mathrm{~nm}$ with 1-nm bandpass filter, as broad as 650-nm bandwidth from 750 to $1400 \mathrm{~nm}$ within $10-\mathrm{dB}$ peak-to-peak flatness is obtained with an output power of $190 \mathrm{~mW}$.

The experimental setup is shown in Figure 5(a). The SC laser source is consisted of a picosecond mode-locked laser oscillator, a spectral filter, two-stage single-mode amplifiers, and 2-mlong PCF with ZDW at $1.02 \mu \mathrm{m}$. The laser oscillator operated in an all-normal-dispersion regime with repetition rate of $20 \mathrm{MHz}$. With $100 \mathrm{~mW}$ pumping power, $25 \mathrm{~mW}$ average output power laser is exported from the $30 \%$ port of the coupler. The pulse duration of highly upchirped pulse was measured to be 10 ps.
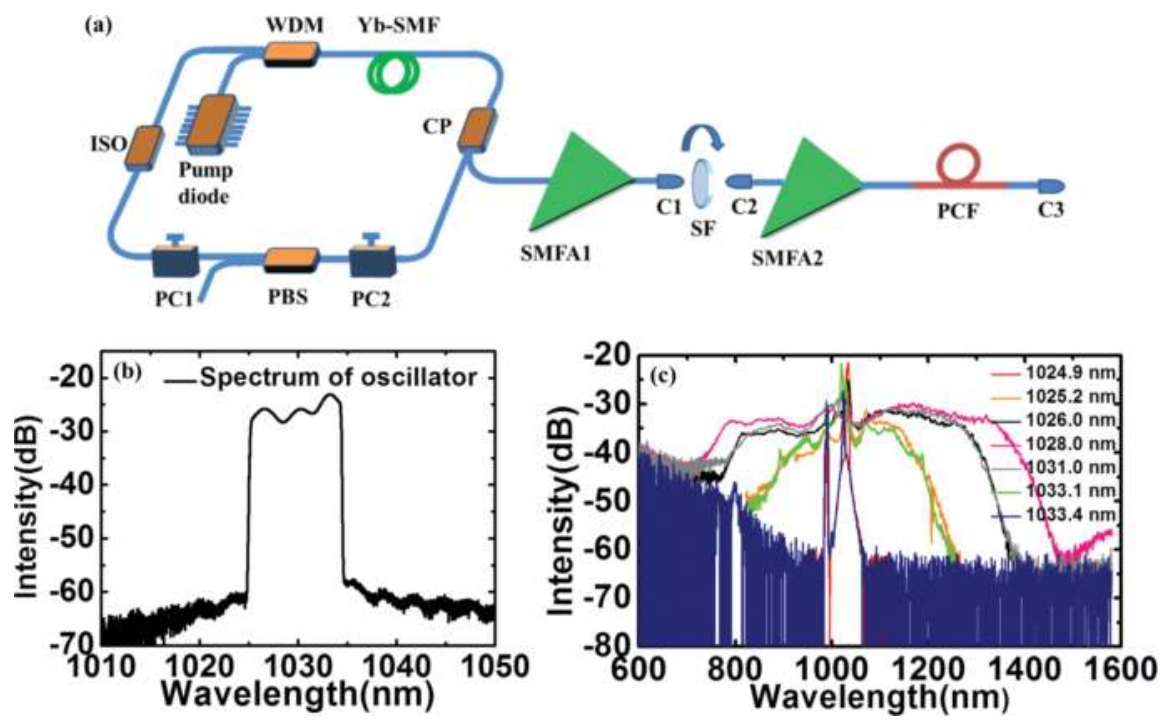

Figure 5. (a) Experimental setup for SC generation. Pump diode: $400 \mathrm{~mW}$ laser diode at $976 \mathrm{~nm}$; WDM: 980/1040 nm wavelength division multiplexer; Yb-SMF: ytterbium-doped single-mode fiber; CP: 30:70 coupler; PC1 and PC2: polarization controller; PBS: polarization beam splitter; ISO: isolator; SMFA1 and SMFA2: single-mode fiber amplifiers; C1, C2, and C3: three collimators; SF: spectral filter; PCF: photonics crystal fiber. (b) The output spectrum of the laser oscillator. (c) SC with different filtering windows.

A bandpass spectral filter with 1-nm bandwidth at $1036 \mathrm{~nm}$ is installed between two singlemode fiber amplifiers. The transparent wavelength of the filter could be tuned from 1024 to $1036 \mathrm{~nm}$ by varying the incident deflection angle. For the large up-chirp with 10-nm spectral width (see Figure 5(b)) and 10-ps temporal duration, corresponding to a time-bandwidth product of 28.3, pulse can be greatly shortened by the filter. The shortest pulse duration of 2.9 ps was obtained with filtering window at $1028 \mathrm{~nm}$. After the second-stage amplifier, the laser pulses could be amplified to an average power up to $190 \mathrm{~mW}$ with $400 \mathrm{~mW}$ pumping power. 
A 2-m length of silica-based PCF with ZDW at $1024 \mathrm{~nm}$ is directly spliced to the fiber end of SMFA2. Figure 5(c) shows the output spectrum with different filtering window. The spectra keep unchanged when the filtering window is at the shoulder of the spectrum, shown as the red curve (1024.9 nm) and blue curve (1033.4 nm) in Figure 5(c). When filtering window is located at the central wavelength of $1028 \mathrm{~nm}$, the 10-dB bandwidth of SC is extended to $650 \mathrm{~nm}$ (from 750 to $1400 \mathrm{~nm}$ ), shown as the pink curve in Figure 5(c). Besides, filtering windows above or below the central wavelength produce a less broad SC.

\subsubsection{One octave supercontinuum for frequency comb generation}

Broadband supercontinuum of bandwidth up to $1250 \mathrm{~nm}$ can also be provided by HNLFs with spectral-tailored femtosecond pump pulses produced by erbium-doped power amplifiers. The schematic diagram of the experiment is shown in Figure 6(a).
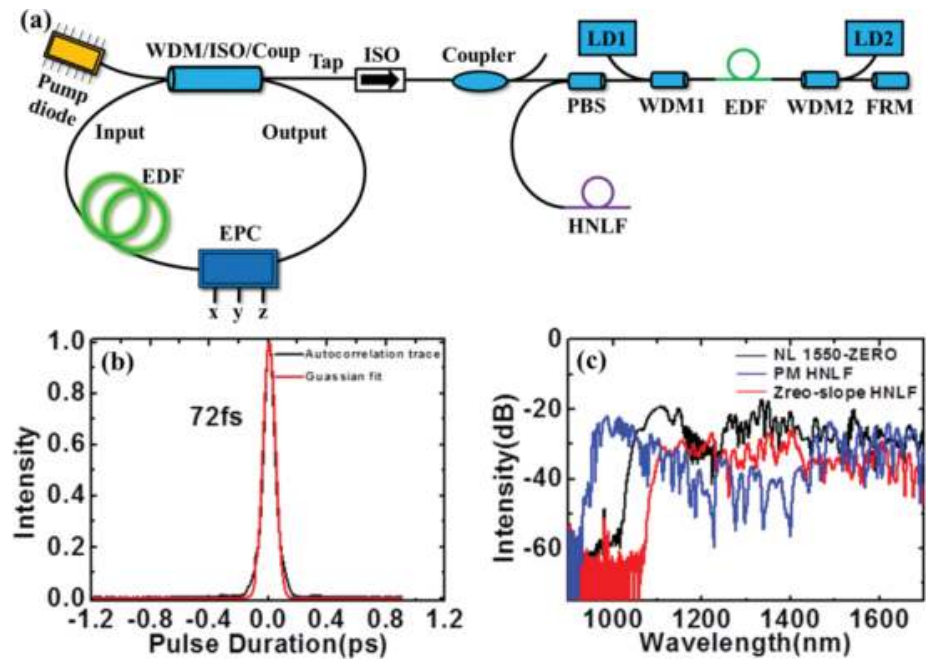

Figure 6. (a) Schematic diagram for SC generation. Pump diode: 400-mW pump at $976 \mathrm{~nm}$; WDM/ISO/Coup: the device combines wavelength division multiplexer, isolator, and coupler; EDF: erbium-doped fiber; EPC: electric polarization controller; ISO: isolator; coupler: 30:70 polarization-maintaining coupler; PBS: polarization beam splitter; HNLF: high nonlinear fiber; LD1 and LD2: pumps at $976 \mathrm{~nm}$; WDM1 and WDM2: 980/1550 nm wavelength division multiplexer; FRM: Faraday rotation mirror. (b) Autocorrelation trace of chirped pulses poured into HNLFs. (c) SC generated by different kinds of HNLFs on logarithmic coordinate.

The laser system consisted of an erbium-doped mode-locking fiber oscillator, a single-mode fiber amplifier (SMFA), and 20-cm-long PM-HNLF. To improve the mode-locking stability, an electric polarization controller (EPC) is utilized to replace the conventional mechanical polarization controller such that automatic and active control of mode-locking is accessible. By applying the voltage on three axes $(x, y$, and $z)$ of EPC, accurate control of the temporal duration, spectral shape, $f_{\text {rep }}$ and $f_{\text {ceo }}$ can be achieved $[47,48]$. 
With the help of a PBS and a FRM, a dual-pass single-mode fiber amplifier with bidirectional pump configuration was used to boost the laser average power to more than $150 \mathrm{~mW}$ average power and reduce the environmental disturbance on SMFA. The pulse duration at the output port was measured to be 2.84 ps. Additional PM-1550 fiber was used to dechirp the preamplified pulse to $72 \mathrm{fs}$ (shown in Figure 6(b)). Therefore, considering a repetition rate of $60 \mathrm{MHz}$, the pulse peak power achieved as high as $34.7 \mathrm{~kW}$. Three types of HNLFs, such as NL 1550-ZERO, PM-HNLF, and Zero-slope HNLF, were applied to generate the supercontinuum by splicing the HNLFs to the dechirping fiber directly.

As shown in Figure 6(c), 20-cm-long PM-HNLF with nonlinearity of $10.5 \mathrm{~W}^{-1} \mathrm{~km}^{-1}$ achieved the broadest spectrum, covering from 950 to $2200 \mathrm{~nm}$, which is sufficient broad to produce $f_{\text {ceo }}$ signal. The HNLF type should be taken into consideration as it influences the SC generation. We used three kinds of HNLFs: 25-cm-long NL 1550-ZERO with nonlinear coefficient of 10.4$\mathrm{W}^{-1} \mathrm{~km}^{-1}$ and effective mode area of $13-\mu \mathrm{m}^{2}, 20-\mathrm{cm}$-long PM-HNLF with nonlinear coefficient of $10.5-\mathrm{W}^{-1} \mathrm{~km}^{-1}$ and effective mode area of $12.7-\mu \mathrm{m}^{2}$, 25-cm-long Zero-slope HNLF with nonlinear coefficient of $10.8-\mathrm{W}^{-1} \mathrm{~km}^{-1}$ and effective mode area of $12.4-\mu \mathrm{m}^{2}$, and the corresponding SC was depicted in Figure 6(c). Obviously, PM-HNLF produces broader spectrum than other two HNLFs.

As shown in Figure 7, a collinear setup was established for the detection of $f_{\text {ceo }}$ signal. The SC generated by $20-\mathrm{cm}$-long PM-HNLF was coupled into free space via a lens (L1) with adjustable focal length. An inline f-2f interferometer, including a PPLN, several wave plates and lens, and a PM-fiber delay line, is used to produce the temporal overlapped components at $1.0 \mu \mathrm{m}$. The long-wavelength component of SC at $2092 \mathrm{~nm}$ was frequency doubled to match with the short-wavelength component at $1046 \mathrm{~nm}$. After the PPLN, two lenses, L3 and L4, were used to couple the two components at $1046 \mathrm{~nm}$ back to PM-980 fiber. A half-wave plate, HWP2, is used to adjust the energy ratio on the fast and slow axes of PM-980 fiber. The pulse transmitted along the slow axis experiences a delay relative to the pulse on fast axis. With an optimized fiber length of $3.4 \mathrm{~m}$, the differential delay between the fast and slow axes could be fully

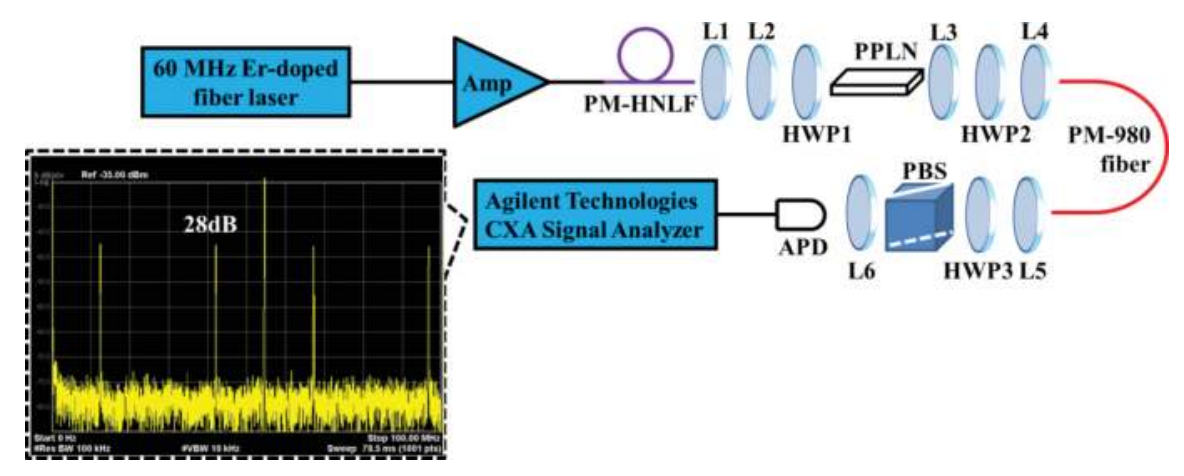

Figure 7. Setup for $f_{\text {ceo }}$ detection. Amp: fiber amplifier; L1, L4, and L5: optical lens with adjustable focal length; L2, L3, and L6: optical lens with focal length of $50 \mathrm{~mm}$; HWP1, HWP2, and HWP3: half-wave plates; PPLN: periodically poled lithium niobate; PBS: polarization beam splitter; APD: avalanche photodiode. 
compensated [49]. Subsequently, a half-wave plate, HWP3, as well as a PBS were used to selected pulses to generate $f_{\text {ceo }}$ signal on APD. Finally, with 28-dB signal-to-noise ratio was generated by using this setup.

\section{Nonlinear fiber amplifier}

With the increasing applications in frequency metrology, $\mathrm{THz}$ generation, and in cataract surgery, the development for high-energy transform-limited pulse generation around $1.55 \mu \mathrm{m}$ is still a fascinating area [50-53]. Owing to the limited available output power from laser oscillator, erbium-doped fiber amplifiers (EDFAs) are commonly used. Nevertheless, high-power amplification in EDFA is inevitably accompanied by several unwanted effects, such as SRS and amplified spontaneous emission (ASE), which would significantly deteriorate the temporal and spectral duration of pulse [54]. Chirped-pulse amplification (CPA) provides an effective way to decrease pulse peak power and avoid the nonlinearity in optical fibers [55-58]. In CPA, strong stretching and compression occurs to extract more energy and avoid nonlinear distortion as well as damage. However, CPA is inevitably accompanied by the gain-narrowing effect and therefore hardly produces pulse with temporal duration less than $400 \mathrm{fs}$ [59].

Even though CPA has many advantages over the other techniques in amplifying pulses around $1.55 \mu \mathrm{m}, \sim 100$-fs pulse duration with above 10-nJ pulse energy is still a challenge because of the spectral gain-narrowing effects and nonlinear phase accumulation. Moreover, due to the involvement of bulk media, CPA is not suited for applications that require compact size and alignment-free laser source. A recent developed technique, divided-pulse amplification (DPA), opens up a new way for high-power laser pulse amplification [60-62].

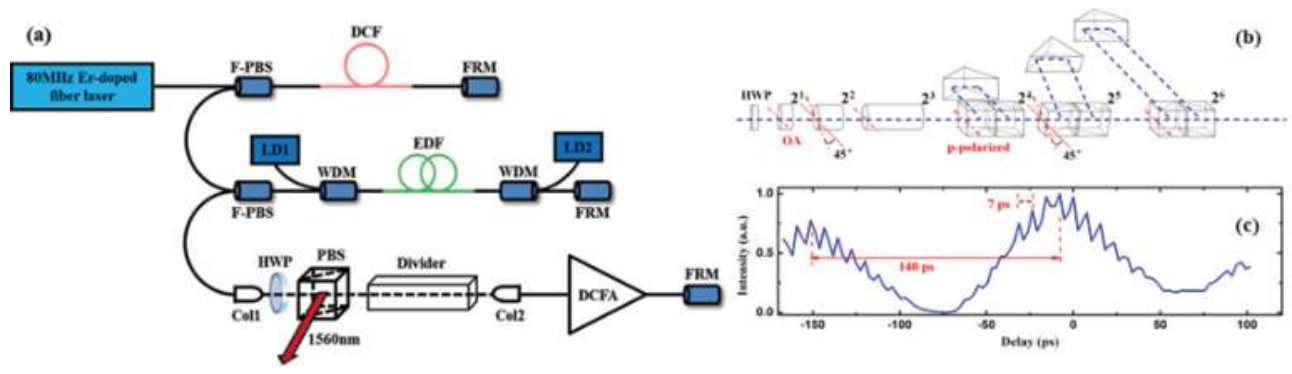

Figure 8. (a) Schematic diagram for laser system. F-PBS: fiber-coupled polarization beam splitter; DCF: dispersion compensation fiber; FRM: Faraday rotation mirror; LD1, LD2: pump diodes at $976 \mathrm{~nm}$; WDM: wavelength division multiplexer; EDF: erbium-doped single-mode fiber; Col1, Col2: high-power collimators; HWP: half-wave plate; PBS: polarization beam splitter cube; DCFA: double-clad fiber amplifier. (b) Schematic of the pulse divider. The left-hand three cylinders $(21,22$, and 23) represent YVO4-based dividers with given direction of crystal optical axes (OAs) shown as the red dash dot lines. The right-hand three parts $(24,25$, and 26) represent PBS-based dividers with p-polarized direction shown as red dash dot lines. The red dot lines represent the horizontal plane, which is the same direction as the OA of 21 and 23 and the p-polarized direction of 24 and 26. (c) The measured autocorrelation trace of the divided replicas. 
In the configuration of DPA, the initial pulse is divided into a sequence of lower-intensity pulse with orthogonal polarization for successive replicas, and subsequently, the low-energy pulse is amplified and then recombined to create a high-energy pulse [61, 63].

In this section, we mainly focus on DPA at $1.56 \mu \mathrm{m}$ where pulse amplification and compression can be simultaneous carried out so that a separate compressor is no longer necessary. The schematic diagram of DPA is shown in Figure 8(a). The experimental setup is composed of a mode-locked fiber laser, a fiber stretcher, a single-mode fiber amplifier for preamplifying, and a pulse-divider as well as a double-clad fiber amplifier for main amplification. The Er-doped fiber laser with $80-\mathrm{MHz}$ repetition rate shared the same configuration as Figure 6(a), which takes the advantage of EPC to actively control the mode-locking. A photodiode and an electric loop were applied to monitor and feedback control the EPC for long-term stable operation. The fiber oscillator consisted of 1.74-m SMF28-e fiber with dispersion parameter of $19 \mathrm{ps} / \mathrm{nm} /$ $\mathrm{km}$ and $0.82-\mathrm{m}$ Er-doped fiber with dispersion parameter of $-51 \mathrm{ps} / \mathrm{nm} / \mathrm{km}$. There, the laser operated in the stretched-pulse regime and produced positively chirped pulses. As a result, with 200-mW pumping power at $976 \mathrm{~nm}$, the laser oscillator produces 5-mW output average power with 1.5-ps pulse duration and 28-nm spectral bandwidth, corresponding to a timebandwidth product of 5.2 .

A fiber stretcher is spliced to the output of the fiber oscillator to stretch laser pulse and control the quantity of frequency up-chirp. However, over-long fiber could inevitably introduce too much high-order dispersion which is hardly compensated by the pulse-compressing stage. For the current configuration, a double-pass fiber stretcher consisting of a fiber-coupled PBS with PM fiber at input/output port and non-PM fiber at common port, a segment of non-PM dispersion compensation fiber with 6.0- $\mu \mathrm{m}$-mode field diameter and $-38 \mathrm{ps} / \mathrm{nm} / \mathrm{km}$ dispersion at $1550 \mathrm{~nm}$, and a FRM is used to reduce the environmental perturbation.

In our experiment, 6-m dispersion compensation fiber was applied to stretch pulses from the fiber oscillator. A dual-pass bidirectionally pumped single-mode fiber preamplifier was used to boost the average power to more than $100 \mathrm{~mW}$ to ensure efficient operation of the subsequent amplifiers. A FRM reflected the incident pulse to suppress ASE noise and rotated the polarization of the pulses by $90^{\circ}$ to cancel all birefringence effects in the dual-pass amplifier.
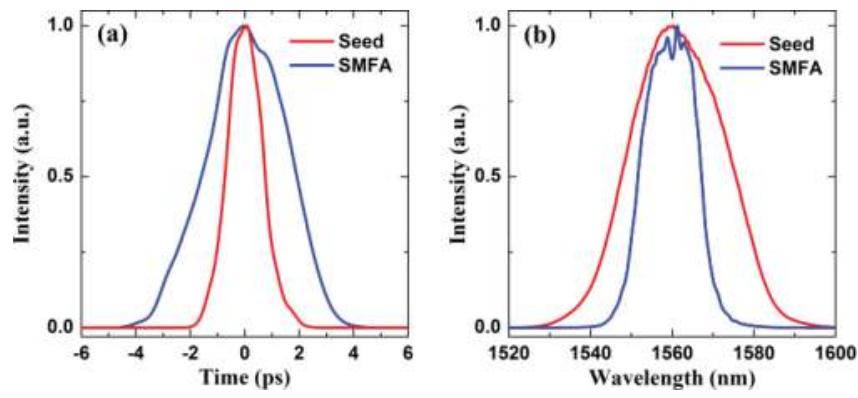

Figure 9. The temporal duration (a) and spectral bandwidth (b) of laser pulses from laser oscillator (red curves) and SMFA (blue curves). 
A fiber-based polarization beam splitter (F-PBS) was used to couple the seed laser to the preamplifier and reflected preamplified pulses to subsequent components. The output characters of the preamplifier were shown as the blue curves in Figure 9(a) and (b). The FWHM temporal duration and spectrum bandwidth of the preamplified pulses is 4 ps and $15 \mathrm{~nm}$, respectively, generating a time-bandwidth product of 7.4. Dramatic decrease in spectral bandwidth was observed due to the limited transmission bandwidth of WDM and FRM as well as spectral-narrowing effect in fiber amplifier.

Then, the concept of DPA was carried out to boost the laser to Watt-level average power. The preamplified laser is coupled into free space by collimator C1 and rotated to horizontal polarization to reach maximum transmission on PBS. The pulse division and combination were achieved by applying cascaded $\mathrm{YVO}_{4}$-based and PBS-based dividers with the help of a FRM to reflect the replicas passing through the same divider but in the opposite direction. Each divider ( $\mathrm{YVO}_{4}$-based or PBS-based) can divide a single pulse into two cross-polarized replicas; hence, a single seed pulse could be temporally divided into $2^{N}$ (where $N$ is the stage number of the divider) replicas. Ideally, each replica has identical pulse energy after division. As depicted in Figure 8(b), three $\mathrm{YVO}_{4}$ crystals with lengths of 10, 20, and $40 \mathrm{~mm}$ divided the initial pulse into $N=8$ replicas. A half-wave plate (HWP) was used to produce the desired polarization of input pulses. The first $\left(2^{1}\right)$ and third $\left(2^{3}\right) \mathrm{YVO}_{4}$ crystals had their crystal optical axes (OA) oriented in the same direction as the horizontal plane, while the OA of the second $\left(2^{2}\right) \mathrm{YVO}_{4}$ crystal oriented at a $45^{\circ}$ angle to the horizontal plane. The polarization-mode delay between ordinary and extraordinary waves in $\mathrm{YVO}_{4}$ is $0.7 \mathrm{ps} / \mathrm{mm}$ at $1560 \mathrm{~nm}$. The shortest crystal length for our system was chosen to split the input pulse into replicas with 7 ps separation, about twice of the seed pulse duration.

To mitigate the nonlinearity in main amplifier, the string of pulse $(N=8)$ was further divided by three PBS-based dividers, resulting in a final pulse number of 64 . For PBS-based divider, each incoming pulse was divided into an s-polarized beam and a p-polarized beam. All ppolarized components were directly transmitted the PBS, while the s-polarized components were reflected to the folded delay line. For the sake of simplicity, the second PBS-based divider $\left(2^{5}\right)$ had its p-polarized direction oriented $45^{\circ}$ to the direction of the horizontal plane, while the first $\left(2^{4}\right)$ and third $\left(2^{6}\right)$ PBS-based oriented in the same direction as the horizontal plane, such that separate half-wave plates were no longer necessary.

Owing to the delay length of $10,20,40,26.8,53.6$, and $107.2 \mathrm{~mm}$, the $2^{1}, 2^{2}, 2^{3}, 2^{4}, 2^{5}$, and $2^{6}$ stages approximately provided time delay of $7,14,28,130,260$, and 520 ps, respectively. Figure 8(c) shows the measured autocorrelation trace of the pulse string which matches well with the designed time delays. The 7-ps interval between adjacent peaks in the same envelope was consistent with the expected time delay with 10-mm increment length of $\mathrm{YVO}_{4}$, and the 140 -ps spacing between two adjacent envelopes was consistent with the expected time delay introduced by the PBS-based divider.

Intuitively, for simultaneous pulse amplification and compression in EDFAs, a positively prechirping seed pulse is desired. Numerical simulations show that there exists an equilibrium position that can not only restrict excessive nonlinear effects to ensure high-quality temporal integrity but also produce sufficient optical nonlinearity to broaden the spectrum around the 
wavelength of $1.55 \mu \mathrm{m}$. The generalized nonlinear Schrödinger equation (7) with the split-step Fourier method was used to carry out the simulation [24].

$$
\frac{\partial A}{\partial z}=\frac{\alpha}{2} A+\sum_{n \geq 2} \frac{i^{n+1}}{n !} \beta_{n} \frac{\partial^{n} A}{\partial T^{n}}+i \gamma A \int_{-\infty}^{\infty} R\left(T^{\prime}\right)\left|A\left(z, T-T^{\prime}\right)\right|^{2} d T^{\prime}
$$

where $A=A(z, t)$ is the complex amplitude of the pulse envelope of pulses, $\alpha$ is the laser gain coefficient, $\beta_{n}$ is the dispersion parameter at $\omega_{0}(1560 \mathrm{~nm})$, and $\gamma\left(3 \mathrm{~W}^{-1} \mathrm{~km}^{-1}\right)$ is the nonlinear coefficient. The right-hand side of Eq. (7) models laser gain, dispersion, and nonlinearity. Pulse of 2.5-ps temporal duration and 19.9-nm spectral width (corresponding to $0.16 \mathrm{ps}^{2}$ prechirping on 180-fs transform-limited pulse) and a pulse energy of $0.05 \mathrm{~nJ}$ were applied in the simulation.
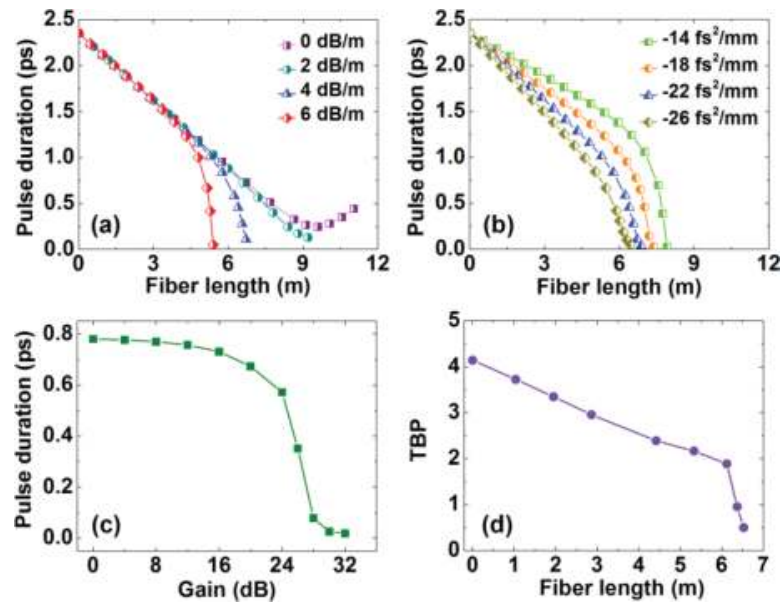

Figure 10. Amplified output pulse duration versus propagation length for the cases of different $\alpha$ (a) and $\beta_{2}$ (b). (c) Pulse duration versus the total gain provided by 6.5 -m fiber. (d) Time-bandwidth product at different position of gain fiber.

The interplay of the SPM and group-velocity dispersion (GVD) as well as laser gain can lead to a qualitatively different behavior compared with that expected from them alone. SPM broadened the spectrum with increase in pulse energy, and simultaneously, the anomalous dispersion of the fiber compressed the new spectral components resulting in temporal shortening. Figure 10(a) compares the simulation results with different $\alpha$ but a fixed $\beta_{2}\left(-22 \mathrm{fs}^{2} /\right.$ $\mathrm{mm})$. It is clear that pulse compression operates in linear regime when the laser gain is low, then it enters in nonlinear regime when the laser gain gradually increased. The shortest transform-limited pulse duration decreased from $180 \mathrm{fs}$ at $7.0 \mathrm{~m}(\alpha=0 \mathrm{~dB} / \mathrm{m})$ to $60 \mathrm{fs}$ at $4.3 \mathrm{~m}$ $(\alpha=3 \mathrm{~dB} / \mathrm{m})$. Figure 10(b) compares the pulse compression with different $\beta_{2}$ but a fixed $\alpha$ 
$(3 \mathrm{~dB} / \mathrm{m})$. The maximum pulse energies with respect to fiber length of $4.65,5.07$, and $5.53 \mathrm{~m}$ reached 1.24, 1.66, and $2.28 \mathrm{~nJ}$, respectively. Therefore, higher $\alpha$ and smaller $\left|\beta_{2}\right|$ are benefit to overcome spectral bandwidth limitation for high-energy pulse amplification. For reference, the blue curves in Figure 10(a) and (b) present pulse evolution with the same parameters.

Next, we focus on pulse amplification and compression in a fixed fiber length by way of guiding the subsequent experiment. About 5.0-m-long fiber with $\beta_{2}=-22 \mathrm{fs}^{2} / \mathrm{mm}$ was introduced to simulate the output pulse duration and the time-bandwidth product (TBP) at different position along the fiber. As shown in Figure 10(c), when the total gain is smaller than $16 \mathrm{~dB}$, the output pulse duration deceases linearly owing to the GVD and insufficient nonlinearity. As the total gain is greater than $24 \mathrm{~dB}$, the output pulse duration dramatically decreases owing to strong nonlinear compression. Theoretically, pulse as short as $80-\mathrm{fs}$ duration can be achieved with a total gain of nearly $28 \mathrm{~dB}$. Although the pulse duration could be further decreased to $20 \mathrm{fs}$ with $32-\mathrm{dB}$ gain, considerable pedestal as well as wave breaking appears due to excessive nonlinearity. Meanwhile, the TBP of the pulse along the fiber gradually decreases from 4.1 at the input port to 0.5 at the output port.

Experimentally, the divider first operated with $\times 8$ replicas. A dual-pass double-cladding fiber amplifier (DCFA) was used to boost the divided pulses. The DCFA consisted of 1.2-m 12/130 Er-doped double-clad fiber and 2.5-m SMF fiber. With a pump power of $4.3 \mathrm{~W}$ at $976 \mathrm{~nm}$, the DCFA delivered $600 \mathrm{~mW}$ output power at $1560 \mathrm{~nm}$, as measured at PBS output port. Along the first pass of DCFA, pulse evolution worked in the near-linear regime. Subsequently, as the pulse reflected by the FRM and passed through DCFA again, SPM and anomalous dispersion brought the pulse amplification into the moderate nonlinearity regime. Figure 11(a) and (b) shows pulse duration and spectral bandwidth of the combined pulse measured by an FROG (15-100-USB, Swamp Optics). The linear spectral phase indicates a nearly transformlimited pulse with the FWHM duration of $97 \mathrm{fs}$ if a Gaussian pulse shape is assumed. The output power limitation was resulted from splicing losses of different type fibers and the insertion loss of FRM.
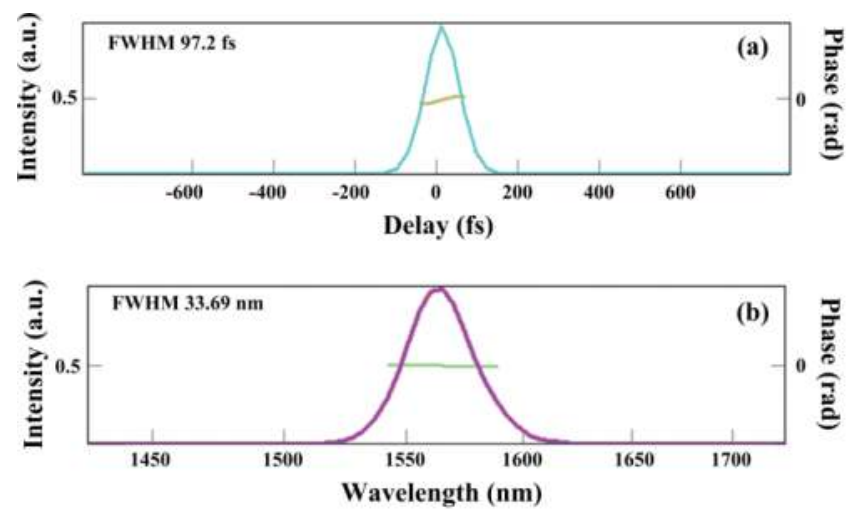
Figure 11. The measured pulse duration (a) and spectral width (b) of pulses from the nonlinear fiber amplifier.

Furthermore, a PPLN with 20.9- $\mu$ m poling period and 0.3-mm length was used for frequency-doubling the amplified laser and checking the available peak power at $1560 \mathrm{~nm}$. A pair of lens was used to focus and collimate the input and output beam on the PPLN, respectively. The output average power at $1560 \mathrm{~nm}$ and the corresponding SHG is shown in Figure 12. The highest SHG conversion efficiency was obtained as 56.3\% with $302 \mathrm{~mW}$ incident power at $1560 \mathrm{~nm}$. Further increasing the power at $1560 \mathrm{~nm}$ induced decay of conversion efficiency, shown as the black squares in Figure 12.

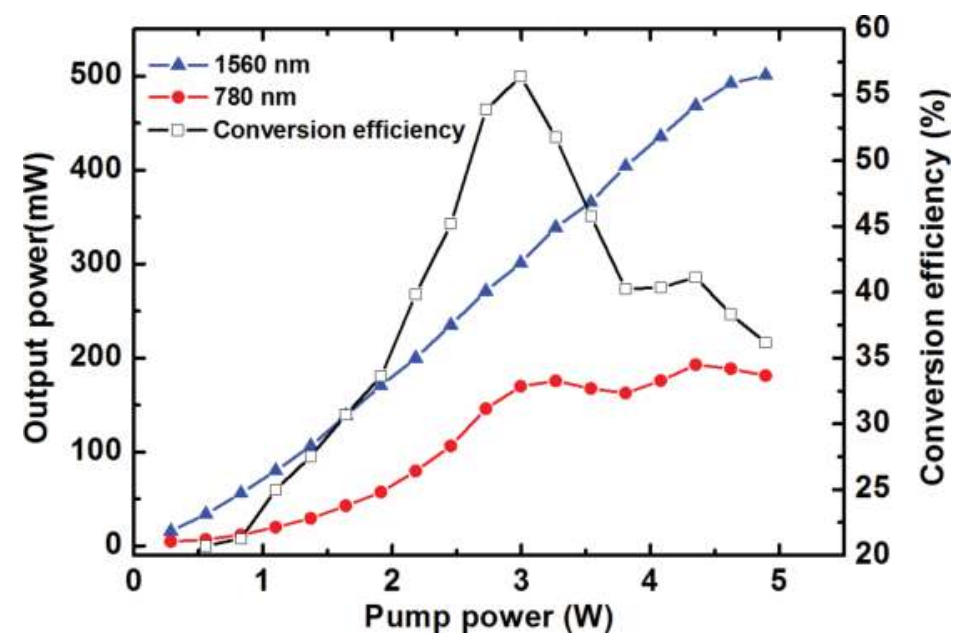

Figure 12. The output average power of $1560 \mathrm{~nm}$ (blue triangles) and $780 \mathrm{~nm}$ (red circles), and the SHG conversion efficiency.

To extract more energy from the double-pass amplifier, we will increase the number of the replicas from 8 to 16 and 32 . The results for $\times 16$ and $\times 32$ replicas are still under investigation.

In conclusion, a divided fiber laser fiber amplifier delivering $500 \mathrm{~mW}$ average power at $1560 \mathrm{~nm}$ by the interplay between divided prechirped pulse amplification and nonlinear pulse compression. A small core double-clad erbium-doped fiber with anomalous dispersion carries out the pulse amplification and simultaneously compresses the laser pulses such that a separate compressor is no longer necessary. A numeric simulation reveals the existence of an optimum fiber length for producing a transform-limited pulse. Furthermore, frequency doubling to $780 \mathrm{~nm}$ with $170-\mathrm{mW}$ average power is realized by using a PPLN at room temperature. 


\section{Repetition rate stabilization}

Fiber-based frequency comb is recognized as the key breakthrough in the field of optics for it brings high accuracy in frequency domain as well as low jitter in time domain [49, 64-67]. Principally, as a frequency comb, two RF frequencies, $f_{\text {ceo }}$ and $f_{\text {rep }}$, are required to be stabilized to external references. Therefore, the optical frequencies can be written as $v=m \times f_{\text {rep }}+f_{\text {ceo }}$ where $m$ is a large integer of order $10^{6}$ that indexes the comb line. Nevertheless, recent developments in adaptive dual-comb spectroscopy successfully employed free-running mode-locked lasers where the $f_{\text {ceo }}$ instabilities could be compensated by data acquisition and electronic signal processing $[68,69]$. Therefore, high accuracy $f_{\text {rep }}$ stabilization of passively ML lasers is of great importance.

The relatively mature method for $f_{\text {rep }}$ locking is to use a piezoelectric ceramic transducer (PZT) to control the geometrical length $\mathrm{L}$ of the laser cavity, and the best locking accuracy is in the range of $\pm 0.5 \mathrm{mHz}$ with the corresponding SD of $220 \mu \mathrm{Hz}$ [70]. However, the PZT-based stabilization encounters many limitations, such as significant positioning errors, hysteresis effect, bulky-design, and the need for time-consuming alignment.

In this section, we focus on the $f_{\text {rep }}$ stabilization by using optical pumping scheme which can be achieved via resonantly enhanced optical nonlinearity or so-called pump-induced refractive index change (RIC) in doped fibers. In optical pumping scheme, the $f_{\text {rep }}$ is stabilized by modulating the refractive index $n$, while keeping the geometrical cavity length $L$ fixed. In the past, this method has been successfully applied in fiber switch where a low pump power and a short length doped fiber are sufficient for the switching [71]. Moreover, the validity of this concept has also been achieved in coherent combining and adaptive interferometry [72]. In 2013, Rieger et al. reported all-optical stabilized repetition rate by using the RIC-based method. With the help of thermos-electric element, over 12-h long-term stabilization was achieved in an NPE-mode-locked Er-doped fiber laser, while the SD of repetition rate drift was measured to be $22 \mathrm{mHz}$. A recent experiment extends this concept to $\mathrm{Yb}$-fiber laser and achieves $1.39-\mathrm{mHz}$ SD of residual fluctuation in an hour measurement [73].

As reported in Ref. [74], a commercial available pump current supply can provide a minimum resolution of pump power as $1.5 \mu \mathrm{W}$ and thus achieve a controlling accuracy of $0.05 \mathrm{~Hz}$, which is more than two orders of magnitude than PZT-based method. Therefore, an interesting experiment worth to do is to use RIC-method to achieve high-precision $f_{\text {rep }}$ stabilization. So far, the RIC method has been fully investigated in NPR mode-locked lasers, which applied non-PM fibers and components [73-75], and the locking accuracy limited to $\sim \mathrm{mHz}$. Considering the environmental perturbation on non-PM fiber, a straightforward idea is to implement RIC method in a PM fiber laser. Therefore, the following part will discuss high-precision repetition rate stabilization by using RIC method in a PM figure-eight laser cavity.

The laser setup shown in Figure 13 is same as Figure 4(a), except the net dispersion of laser cavity. In the current experiment setup for all-optical repetition stabilization, a 56-cm-long $\mathrm{Er}^{3+}-$ doped fiber (EDF2) is spliced asymmetrically in the NALM to act as a frequency controller, while the LD3, which is controlled by the error signal from frequency mixer, provides the 
feedback modulating pump power via WDM3 on EDF2. Besides, a segment of DCF38 is used to compensate the anomalous dispersion of PM1550 fiber. The dispersion of linear loop and the NALM was estimated numerically to be -0.208 and $0.025 \mathrm{ps}^{2}$, producing $-0.183 \mathrm{ps}^{2}$ net dispersion for the whole cavity. Self-started mode-locking in multiple-pulse regime can be achieved by over-pumping method, and stable single-pulse operation can be obtained by decreasing the pump power of LD1 and LD2. At fundamental repetition rate of $11.9 \mathrm{MHz}$, the figure-eight laser cavity delivers $1.5-\mathrm{mW}$ average power via CP2.

The repetition rate was detected by PD3 and successively compared with standard reference ( $\mathrm{Rb}$ clock) in a frequency mixer to produce the error signal. Subsequently, the error signal was filtered and amplified by low-noise voltage preamplifier with frequency cutoff at $1 \mathrm{MHz}$ and a maximum voltage gain of $5 \times 10^{4}$ and further processed by a proportional-integral-derivative (PID) controller.

The long-term stabilization was depicted in Figure 14. As low as $27-\mu \mathrm{Hz}$ accuracy is achieved within 16-h measurement. The inset of Figure 14 magnifies the measured dates from 30,000 to $31,000 \mathrm{~s}$ and shows fluctuation range within $\pm 0.1 \mathrm{mHz}$. Typically, thermal effect, Kerr nonlinear effect, pump-induced nonlinear effect, and random acoustic perturbations contribute to the precision of $f_{\text {rep }}$ stabilization. For our experiment, a temperature-controlled incuba-

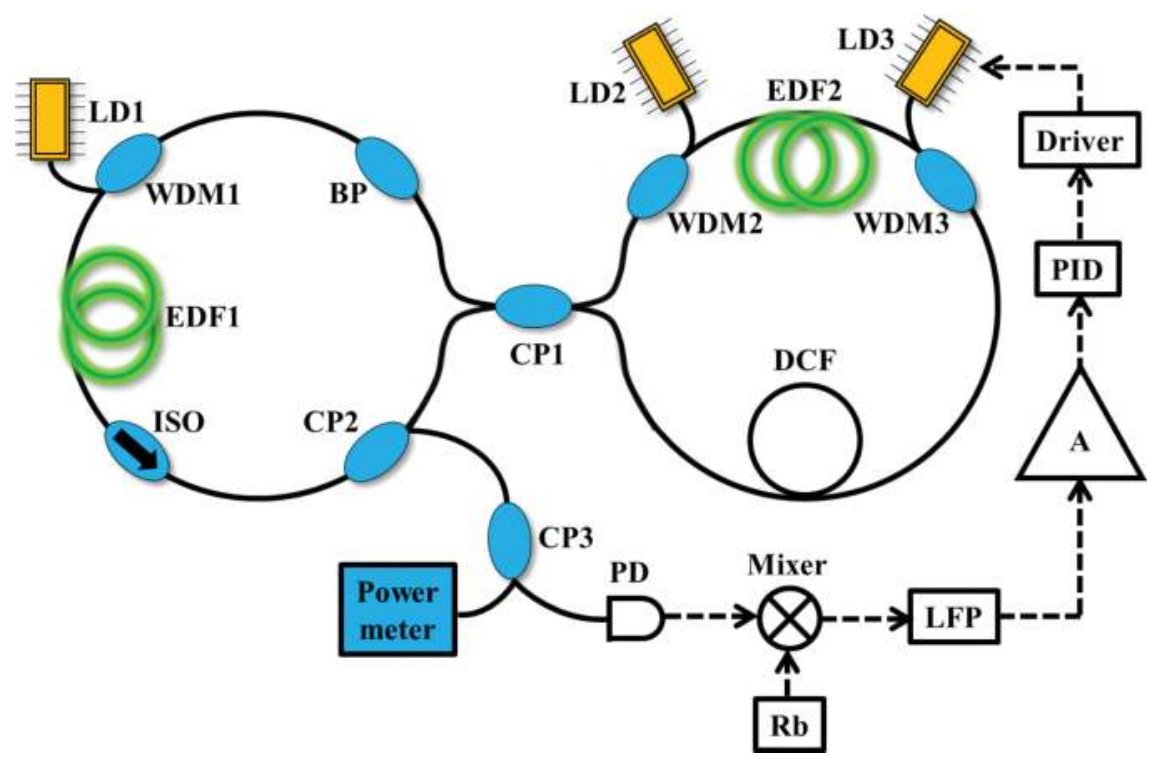

Figure 13. Experimental setup. LD1, LD2, and LD3: pump diodes at $976 \mathrm{~nm}$; WDM1, WDM2, and WDM3: 980/1550 nm wavelength division multiplexers; EDF1, EDF2: erbium-doped fiber; BP: 2-nm bandpass filter centered at $1550 \mathrm{~nm}$; CP1, CP2, and CP3: 1550 nm couplers with splitting ratio of 45:55, 30:70, and 50:50, respectively; DCF: dispersion compensation fiber; PD: photodiode detector; Rb: Rubidium clock; LFP: low-pass filter; A: electronic amplifier; PID: proportional-integral-derivative controller; Driver: precision current source. 
tor with a ripple of $0.2^{\circ} \mathrm{C}$ was used to take the laser cavity to isolate environmental perturbation. As for Kerr-nonlinearity, the RIC is proportional to the traveling power of resonant laser. Assuming 5-mW traveling power in NALM, the Kerr-induced RIC is estimated as $1.2 \times 10^{-}$ $7 / \mathrm{mW}$, having the same order of magnitude of the pump-induced RIC $\left(2.1 \times 10^{-7} / \mathrm{mW}\right)$. However, when the pump power of LD3 increased from 30 to $205 \mathrm{~mW}$, only 1.6\% of output power change was observed, which means little change on the dynamic process of pulse evolution in NALM. Thus, the Kerr-induced RIC is near $\sim 1 \%$ of the RIC by pump-induced nonlinearity. Therefore, we postulate that the nonlinearity on the RIC of fibers owes to pumpinduced nonlinear effect and thermal effect rather than Kerr effect.

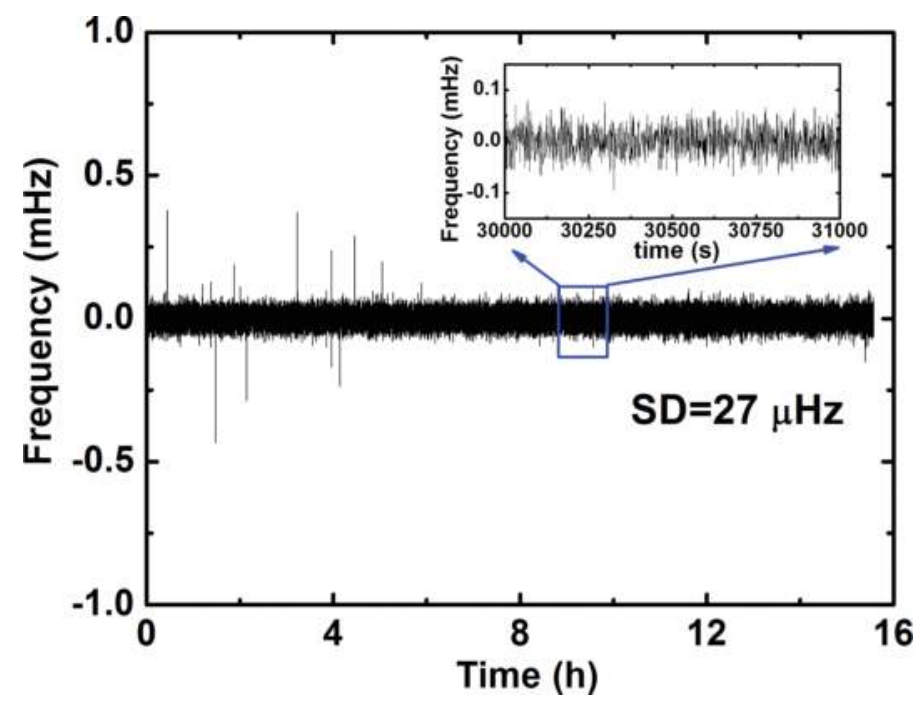

Figure 14. The long-time stabilization of repetition rate.

\section{Conclusion}

In this chapter, we first present several types of mode-locked fiber lasers, as well as their derivatives for SC generation. Second, an effective method named DPA was applied in Erdoped fiber laser system allowing simultaneous pulse amplification and compression so that additional pulse compressor is no longer needed. With $\times 8$ replicas in DPA, as high as $500-\mathrm{mW}$ average power was achieved and the highest SHG conversion efficiency was measured to be $56.3 \%$. Third, an all-optical method, named as pump-induced nonlinearity, is applied to stabilize the repetition rate of a figure-eight Er-doped fiber laser, achieving as low as $27-\mu \mathrm{Hz}$ accuracy within 16-h measurement. 


\section{Author details}

Qiang Hao, Tingting Liu and Heping Zeng*

*Address all correspondence to: hpzeng@phy.ecnu.edu.cn

Shanghai Key Laboratory of Modern Optical System, Engineering Research Center of Optical Instrument and System (Ministry of Education), School of Optical-Electrical and Computer Engineering, University of Shanghai for Science and Technology, Shanghai, China

\section{References}

[1] Hao, Q.; Li, W. \& Zeng, H. (2007). Double-clad fiber amplifier for broadband tunable ytterbium-doped oxyorthosilicates lasers, Opt. Express, Vol. 15(25), 16754-16759.

[2] Xu, J.; Wu, S.; Liu, J.; Li, Y.; Ren, J.; Yang, Q. \& Wang, P. (2014). All-polarizationmaintaining femtosecond fiber lasers using graphene oxide saturable absorber, IEEE Photon. Technol. Lett., Vol. 26(4), 346-348.

[3] Albert, A.; Couderc, V.; Lefort, L. \& Barthelemy, A. (2004). High-energy femtosecond pulses from an ytterbium-doped fiber laser with a new cavity design, IEEE Photon. Technol. Lett., Vol. 16(2), 416-418.

[4] Morin, F.; Druon, F.; Hanna, M. \& Georges, P. (2009). Microjoule femtosecond fiber laser at 1.6 microm for corneal surgery applications, Opt. Lett., Vol. 34(13), 1991-1993.

[5] Kieu, K.; Mehravar, S.; Gowda, R.; Norwood, R. A. \& Peyghambarian, N. (2013). Labelfree multi-photon imaging using a compact femtosecond fiber laser mode-locked by carbon nanotube saturable absorber, Biomed. Opt. Express, Vol. 4(10), 2187.

[6] Li, W.; Hao, Q.; Yan, M. \& Zeng, H. (2009). Tunable flat-top nanosecond fiber laser oscillator and $280 \mathrm{~W}$ average power nanosecond $\mathrm{Yb}$-doped fiber amplifier, Opt. Express, Vol. 17(12), 10113-10118.

[7] Xu, C. \& Wise, F. W. (2013). Recent advances in fiber lasers for nonlinear microscopy, Nat. Photonics, Vol. 7(11), 875-882.

[8] Shi, W.; Fang, Q.; Zhu, X.; Norwood, R. A. \& Peyghambarian, N. (2014). Fiber lasers and their applications [Invited], Appl. Opt., Vol. 53(28), 6554-6568.

[9] Yang, K.; Li, W.; Yan, M.; Shen, X.; Zhao, J. \& Zeng, H. (2012). High-power ultrabroadband frequency comb from ultraviolet to infrared by high-power fiber amplifiers, Opt. Express, Vol. 20(12), 12899-12905. 
[10] Dudley, J. M. \& Coen, S. (2002). Coherence properties of supercontinuum spectra generated in photonic crystal and tapered optical fibers, Opt. Lett., Vol. 27(13), 1180 1182.

[11] Wan, P.; Yang, L. \& Liu, J. (2013). All fiber-based Yb-doped high energy, high power femtosecond fiber lasers, Opt. Express, Vol. 21(24), 29854.

[12] Nicholson, J. W. \& Andrejco, M. (2006). A polarization maintaining, dispersion managed, femtosecond figure-eight fiber laser, Opt. Express, Vol. 14(18), 8160-8167.

[13] Baumgartl, M.; Ortaç, B.; Limpert, J. \& Tünnermann, A. (2012). Impact of dispersion on pulse dynamics in chirped-pulse fiber lasers, Appl. Phys. B, Vol. 107(2), 263-274.

[14] Iii, I. N. (1991). All-fiber ring soliton laser mode locked with a nonlinear mirror, Opt. Lett., Vol. 16(8), 539-541.

[15] Tamura, K.; Ippen, E. P.; Haus, H. A. \& Nelson, L. E. (1993). 77-fs pulse generation from a stretched-pulse mode-locked all-fiber ring laser, Opt. Lett., Vol. 18(13), 1080.

[16] Tamura, K.; Doerr, C. R.; Nelson, L. E.; Haus, H. A. \& Ippen, E. P. (1994). Technique for obtaining high-energy ultrashort pulses from an additive-pulse mode-locked erbiumdoped fiber ring laser, Opt. Lett., Vol. 19(1), 46-48.

[17] Herda, R. \& Okhotnikov, O. G. (2004). Dispersion compensation-free fiber laser modelocked and stabilized by high-contrast saturable absorber mirror, IEEE J. Quantum Elect., Vol. 40(7), 893-899.

[18] Runge, A. F. J.; Aguergaray, C.; Provo, R.; Erkintalo, M. \& Broderick, N. G. R. (2014). All-normal dispersion fiber lasers mode-locked with a nonlinear amplifying loop mirror, Opt. Fiber Technol., Vol. 20(6), 657-665.

[19] Oktem, B.; Ulgudur, C. \& Ilday, F. O. (2010). Soliton-similariton fibre laser, Nat. Photon., Vol. 4(5), 307-311.

[20] Abdelalim, M. A.; Logvin, Y.; Khalil, D. A. \& Anis, H. (2009). Properties and stability limits of an optimized mode-locked Yb-doped femtosecond fiber laser, Opt. Express, Vol. 17(4), 2264-2279.

[21] Zhao, L. M.; Tang, D. Y.; Cheng, T. H.; Tam, H. Y. \& Lu, C. (2007). Bound states of dispersion-managed solitons in a fiber laser at near zero dispersion, Appl. Opt., Vol. 46(21), 4768-4773.

[22] Chong, A.; Renninger, W. H. \& Wise, F. W. (2007). All-normal-dispersion femtosecond fiber laser with pulse energy above 20 nJ, Opt. Lett., Vol. 32(16), 2408-2410.

[23] Erkintalo, M.; Aguergaray, C.; Runge, A. \& Broderick, N. G. (2012). Environmentally stable all-PM all-fiber giant chirp oscillator, Opt. Express, Vol. 20(20), 22669-22674.

[24] Agrawal, G. P. (2001). Applications of nonlinear fiber optics (1st edn): Academic Press; 525 B Street, Suite 1900, San Diego, California 92101-4495, USA. 
[25] Senoo, Y.; Nishizawa, N.; Sakakibara, Y.; Sumimura, K.; Itoga, E.; Kataura, H. \& Itoh, K. (2010). Ultralow-repetition-rate, high-energy, polarization-maintaining, Er-doped, ultrashort-pulse fiber laser using single-wall-carbon-nanotube saturable absorber, Opt. Express, Vol. 18(20), 20673-20680.

[26] Hofer, M.; Fermann, M. E.; Galvanauskas, A.; Harter, D. \& Windeler, R. S. (1998). Highpower 100-fs pulse generation by frequency doubling of an erbium ytterbium-fiber master oscillator power amplifier, Opt. Lett., Vol. 23(23), 1840-1842.

[27] Takayanagi, J.; Kanamori, S.; Suizu, K.; Yamashita, M.; Ouchi, T.; Kasai, S.; Ohtake, H.; Uchida, H.; Nishizawa, N. \& Kawase, K. (2008). Generation and detection of broadband coherent terahertz radiation using 17-fs ultrashort pulse fiber laser, Opt. Express, Vol. 16(17), 12859-12865.

[28] Gaponov, D. A.; Kotov, L. V.; Likhachev, M. E.; Bubnov, M. M.; Cabasse, A.; Oudar, J. L.; Fevrier, S.; Lipatov, D. S.; Vechkanov, N. N.; Guryanov, A. N. \& Martel, G. (2012). High power all-fibered femtosecond master oscillator power amplifier at $1.56 \mu \mathrm{m}, \mathrm{Opt}$. Lett., Vol. 37(15), 3186-3188.

[29] Tang, D. Y.; Zhao, L. M.; Zhao, B. \& Liu, A. Q. (2005). Mechanism of multisoliton formation and soliton energy quantization in passively mode-locked fiber lasers, Phys. Rev. A, Vol. 72(4), 043816.

[30] Takayanagi, J.; Nishizawa, N.; Nagai, H.; Yoshida, M. \& Goto, T. (2005). Generation of high-power femtosecond pulse and octave-spanning ultrabroad supercontinuum using all-fiber system, IEEE Photonic. Technol. Lett., Vol. 17(1), 37-39.

[31] Chen, Y.; Räikkönen, E.; Kaasalainen, S.; Suomalainen, J.; Hakala, T.; Hyyppä, J. \& Chen, R. (2010). Two-channel hyperspectral LiDAR with a supercontinuum laser source, Sensors, Vol. 10(7), 7057-7066.

[32] Kaminski, C. F.; Watt, R. S.; Elder, A. D.; Frank, J. H. \& Hult, J. (2008). Supercontinuum radiation for applications in chemical sensing and microscopy, Appl. Phys. B, Vol. 92(3), 367-378.

[33] Rulkov, A.; Vyatkin, M.; Popov, S.; Taylor, J. \& Gapontsev, V. (2005). High brightness picosecond all-fiber generation in 525-1800 nm range with picosecond $\mathrm{Yb}$ pumping, Opt. Express, Vol. 13(2), 377-381.

[34] Hao, Q.; Guo, Z.; Liu, Y.; Li, W.; Zhang, Q. \& Zeng, H. (2014). Spectrally tailored supercontinuum generation from single-mode-fiber amplifiers, Appl. Phys. Lett., Vol. 104(20), 201112.

[35] Tao, Z.; Yan, W.; Liu, L.; Li, L.; Oda, S.; Hoshida, T. \& Rasmussen, J. C. (2011). Simple fiber model for determination of XPM effects, J. Lightwave Technol., Vol. 29(7), 974-986.

[36] Kudlinski, A.; Pureur, V.; Bouwmans, G. \& Mussot, A. (2008). Experimental investigation of combined four-wave mixing and Raman effect in the normal dispersion regime of a photonic crystal fiber, Opt. Lett., Vol. 33(21), 2488-2490. 
[37] Nodop, D.; Jauregui, C.; Schimpf, D.; Limpert, J. \& Tünnermann, A. (2009). Efficient high-power generation of visible and mid-infrared light by degenerate four-wavemixing in a large-mode-area photonic-crystal fiber, Opt. Lett., Vol. 34(22), 3499-3501.

[38] Hao, Q. \& Huang, Y. C. (2013). Two-octave polarized supercontinuum generated from a Q-switched laser pumped doubly resonant parametric oscillator, Opt. Lett., Vol. 38(11), 1863-1865.

[39] Brown, W. J.; Kim, S. \& Wax, A. (2014). Noise characterization of supercontinuum sources for low-coherence interferometry applications, J. Opt. Soc. Am. A Opt. Image Sci. Vis., Vol. 31(12), 2703-2710.

[40] Klose, A.; Ycas, G.; Maser, D. L. \& Diddams, S. A. (2014). Tunable, stable source of femtosecond pulses near $2 \mu \mathrm{m}$ via supercontinuum of an erbium mode-locked laser, Opt. Express, Vol. 22(23), 28400.

[41] Wadsworth, W.; Joly, N.; Knight, J.; Birks, T.; Biancalana, F. \& Russell, P. (2004). Supercontinuum and four-wave mixing with Q-switched pulses in endlessly singlemode photonic crystal fibres, Opt. Express, Vol. 12(2), 299-309.

[42] Holdynski, Z.; Napierala, M.; Mergo, P. \& Nasilowski, T. (2015). Experimental investigation of supercontinuum generation in photonic crystal fibers pumped with sub-ns pulses, J. Lightwave Technol., Vol. 33(10), 2106-2110.

[43] Cumberland, B. A.; Travers, J. C.; Popov, S. V. \& Taylor, J. R. (2008). 29 W high power CW supercontinuum source, Opt. Express, Vol. 16(8), 5954-5962.

[44] Qiang, H. \& Heping, Z. (2014). Cascaded four-wave mixing in nonlinear Yb-doped fiber amplifiers, IEEE J. Sel. Top. Quant., Vol. 20(5), 900205.

[45] Chen, K. K.; Alam, S.; Price, J. H. V.; Hayes, J. R.; Lin, D.; Malinowski, A.; Codemard, C.; Ghosh, D.; Pal, M.; Bhadra, S. K. \& Richardson, D. J. (2010). Picosecond fiber MOPA pumped supercontinuum source with $39 \mathrm{~W}$ output power, Opt. Express, Vol. 18(6), $5426-5432$.

[46] Kudlinski, A.; George, A. K.; Knight, J. C.; Travers, J. C.; Rulkov, A. B.; Popov, S. V. \& Taylor, J. R. (2006). Zero-dispersion wavelength decreasing photonic crystal fibers for ultraviolet-extended supercontinuum generation, Opt. Express, Vol. 14(12), 5715-5722.

[47] Shen, X.; He, B.; Zhao, J.; Liu, Y.; Bai, D.; Yang, K.; Wang, C.; Liu, G.; Luo, D.; Liu, F.; Hao, Q.; Li, W. \& Zeng, H. (2015). Repetition rate stabilization of an erbium-doped allfiber laser via opto-mechanical control of the intracavity group velocity, Appl. Phys. Lett., Vol. 106(3), 31117.

[48] Xuling, S.; Wenxue, L.; Ming, Y. \& Heping, Z. (2012). Electronic control of nonlinearpolarization-rotation mode locking in Yb-doped fiber lasers, Opt. Lett., Vol. 37(16), 3426-3428. 
[49] Sinclair, L. C.; Coddington, I.; Swann, W. C.; Rieker, G. B.; Hati, A.; Iwakuni, K. \& Newbury, N. R. (2014). Operation of an optically coherent frequency comb outside the metrology lab, Opt. Express, Vol. 22(6), 6996.

[50] Lee, C.; Chu, S. T.; Little, B. E.; Bland-Hawthorn, J. \& Leon-Saval, S. (2012). Portable frequency combs for optical frequency metrology, Opt. Express, Vol. 20(15), 1667116676.

[51] Reichel, S. \& Zengerle, R. (1999). Effects of nonlinear dispersion in EDFA's on optical communication systems, J. Lightwave Technol., Vol. 17(7), 1152-1157.

[52] Mahran, O. \& Aly, M. H. (2016). Performance characteristics of dual-pumped hybrid EDFA/Raman optical amplifier, Appl. Opt., Vol. 55(1), 22-26.

[53] Ju, H. L.; You, M. C.; Young, G. H.; Haeyang, C.; Sang, H. K. \& Sang, B. L. (2005). A detailed experimental study on single-pump Raman/EDFA hybrid amplifiers: static, dynamic, and system performance comparison, J. Lightwave Technol., Vol. 23(11), 34843493.

[54] Varallyay, Z. \& Jasapara, J. C. (2009). Comparison of amplification in large area fibers using cladding-pump and fundamental-mode core-pump schemes, Opt. Express, Vol. 17(20), 17242-17252.

[55] Eidam, T.; Hanf, S.; Seise, E.; Andersen, T. V.; Gabler, T.; Wirth, C.; Schreiber, T.; Limpert, J. \& Tünnermann, A. (2010). Femtosecond fiber CPA system emitting $830 \mathrm{~W}$ average output power, Opt. Lett., Vol. 35(2), 94-96.

[56] Galvanauskas, A.; Cho, G. C.; Hariharan, A.; Fermann, M. E. \& Harter, D. (2001). Generation of high-energy femtosecond pulses in multimode-core $\mathrm{Yb}$-fiber chirpedpulse amplification systems, Opt. Lett., Vol. 26(12), 935-937.

[57] Ancona, A.; Roser, F.; Rademaker, K.; Limpert, J.; Nolte, S. \& Tünnermann, A. (2008). High speed laser drilling of metals using a high repetition rate, high average power ultrafast fiber CPA system, Opt. Express, Vol. 16(12), 8958-8968.

[58] Kuznetsova, L. \& Wise, F. W. (2007). Scaling of femtosecond Yb-doped fiber amplifiers to tens of microjoule pulse energy via nonlinear chirped pulse amplification, Opt. Lett., Vol. 32(18), 2671-2673.

[59] Sobon, G.; Kaczmarek, P.; Gluszek, A.; Sotor, J. \& Abramski, K. M. (2015). J-level, kHzrepetition rate femtosecond fiber-CPA system at 1555 nm, Opt. Commun., Vol. 347, 812.

[60] Guichard, F.; Hanna, M.; Zaouter, Y.; Papadopoulos, D. N.; Druon, F. \& Georges, P. (2014). Analysis of limitations in divided-pulse nonlinear compression and amplification, IEEE J. Sel. Top. Quant., Vol. 20(5), 619-623. 
[61] Kong, L. J.; Zhao, L. M.; Lefrancois, S.; Ouzounov, D. G.; Yang, C. X. \& Wise, F. W. (2012). Generation of megawatt peak power picosecond pulses from a divided-pulse fiber amplifier, Opt. Lett., Vol. 37(2), 253-255.

[62] Hao, Q.; Zhang, Q.; Sun, T.; Chen, J.; Guo, Z.; Wang, Y.; Guo, Z.; Yang, K. \& Zeng, H. (2015). Divided-pulse nonlinear amplification and simultaneous compression, Appl. Phys. Lett., Vol. 106(10), 101103.

[63] Kienel, M.; Klenke, A.; Eidam, T.; Baumgartl, M.; Jauregui, C.; Limpert, J. \& Tünnermann, A. (2013). Analysis of passively combined divided-pulse amplification as an energy-scaling concept, Opt. Express, Vol. 21(23), 29031.

[64] Cingoz, A.; Yost, D. C.; Allison, T. K.; Ruehl, A.; Fermann, M. E.; Hartl, I. \& Ye, J. (2012). Direct frequency comb spectroscopy in the extreme ultraviolet, Nature, Vol. 482(7383), $68-71$.

[65] Hsieh, Y. D.; Iyonaga, Y.; Sakaguchi, Y.; Yokoyama, S.; Inaba, H.; Minoshima, K.; Hindle, F.; Araki, T. \& Yasui, T. (2014). Spectrally interleaved, comb-mode-resolved spectroscopy using swept dual terahertz combs, Sci. Rep., Vol. 4, 3816.

[66] Heinecke, D. C.; Bartels, A. \& Diddams, S. A. (2011). Offset frequency dynamics and phase noise properties of a self-referenced $10 \mathrm{GHz}$ Ti:sapphire frequency comb, Opt. Express, Vol. 19(19), 18440-18451.

[67] Locke, C. R.; Ivanov, E. N.; Light, P. S.; Benabid, F. \& Luiten, A. N. (2009). Frequency stabilisation of a fibre-laser comb using a novel microstructured fibre, Opt. Express, Vol. 17(7), 5897-5904.

[68] Ideguchi, T.; Poisson, A.; Guelachvili, G.; Picque, N. \& Hansch, T. W. (2014). Adaptive real-time dual-comb spectroscopy, Nat. Commun., Vol. 5, 3375.

[69] Schliesser, A.; Picqué, N. \& Hänsch, T. W. (2012). Mid-infrared frequency combs, Nat. Photon., Vol. 6(7), 440-449.

[70] Zhang, W.; Han, H.; Zhao, Y.; Du, Q. \& Wei, Z. (2009). A 350 MHz Ti:sapphire laser comb based on monolithic scheme and absolute frequency measurement of $729 \mathrm{~nm}$ laser, Opt. Express, Vol. 17(8), 6059-6067.

[71] Pantell, R. H.; Sadowski, R. W.; Digonnet, M. J. \& Shaw, H. J. (1992). Laser-diodepumped nonlinear switch in erbium-doped fiber, Opt. Lett., Vol. 17(14), 1026-1028.

[72] Fotiadi, A. A.; Zakharov, N.; Antipov, O. L. \& Megret, P. (2009). All-fiber coherent combining of Er-doped amplifiers through refractive index control in $\mathrm{Yb}$-doped fibers, Opt. Lett., Vol. 34(22), 3574-3576.

[73] Yang, K.; Hao, Q. \& Zeng, H. (2015). All-optical high-precision repetition rate locking of an Yb-doped fiber laser, IEEE Photon. Technol. Lett., Vol. 27(8), 852-855. 
[74] Bo, N.; Dong, H.; Peng, D. \& Jianye, Z. (2013). Long-term repetition frequency stabilization of passively mode-locked fiber lasers using high-frequency harmonic synchronization, IEEE J. Quantum Electron., Vol. 49(6), 503-510.

[75] Rieger, S.; Hellwig, T.; Walbaum, T. \& Fallnich, C. (2013). Optical repetition rate stabilization of a mode-locked all-fiber laser, Opt. Express, Vol. 21(4), 4889-4895. 